\title{
ORGANIC SOLAR CELLS: PRINCIPLES, MECHANISM AND RECENT DVELOPMENTS
}

\author{
Vivek K. A ${ }^{1}$, G. D. Agrawal ${ }^{2}$ \\ ${ }^{I}$ Student, M.Tech- Energy Engineering, Department of Mechanical Engineering, Malaviya National Institute of \\ Technology, Jaipur, Rajasthan, India \\ ${ }^{2}$ Associate Professor, Department of Mechanical Engineering, Malaviya National Institute of Technology, Jaipur, \\ Rajasthan, India
}

\begin{abstract}
Over the past decade organic solar cells dragged lot of attention and research interest due to its wide potential and advantages such as low cost, made of abundant earth materials, simple manufacturing techniques and ability to incorporate various technologies. Although a lot amount of research and development is required to effectively tackle the foreseen advantages. This paper reviews basic fundamental physics of organic solar cells, working mechanism and recent developments in the field.
\end{abstract}

Keywords: Organic solar cell, Plastic/Polymer solar cell, Organic solar cell mechanism, Organic solar cell physics

\section{INTRODUCTION}

The demand for high efficient and affordable solar photovoltaic technologies had resulted in widespread research and development of various technologies including organic solar cells, dye sensitized solar cells [1], quantum dot solar cells [2] etc. Of all the upcoming alternative organic solar cells possesses distinctive advantages such as low cost, made of abundant earth materials, simple manufacturing techniques and ability to incorporate various other technologies [3]. The current highest reported efficiency of organic solar cells are above $10 \%$ [4] and it is widely regarded that the lower operating efficiency of organic solar cells compared to that of typical silicon solar cells doesn't hinder the commercialization potential of organic cells due to its other advantages [5]. Although many other technical limitations and drawbacks including low stability and lifetime [6], limitations in understanding of basic device physics [7] etc. are to be addressed in the very near future as the technology is on the verge of mass commercialization. This paper reviews basic fundamental physics of organic solar cells, working mechanism and recent developments in the field.

\section{BASIC PRINCIPLES OF ORGANIC PHOTOVOLTAIC DEVICES}

Typically most of the organic compounds are inert for electrical conductivity due to the presence of strong covalent bonds. But this general perception was altered by the discovery of conducting polymers by H. Shirakawa, Alan G. M. and Alan J. Heeger in 1977 [8]. In their experiment, trans-polyacetylene was exposed to chlorine, bromine or iodine vapour, which resulted in increase in conductivity of polyacetylene films. This discovery opened up a recently new and wide range of applications including organic displays, organic LEDs, organic and micro-electronics and organic photovoltaics.

Whatever the organic semiconductors be, such as macro molecule dyes, dendrimers, oligomers, polymers etc. all are based on conjugated $\pi$ electron system. A conjugated system is an alteration between the single and double bonds. The important property related to this conjugation are that the $\pi$ electrons are more mobile than $\sigma$ electrons. Therefore by absorption of energy as in case of organic solar cells or by absorbing electrical energy in case of organic LEDs or displays, the $\pi$ bonds system breaks creating excitons or free charges or emission of light. Molecular $\pi-\pi^{*}$ orbitals corresponds to the Highest Occupied Molecular Orbital (HOMO) and Lowest Unoccupied Molecular Orbital (LUMO). In a crystalline semiconductor these corresponds to conduction band (CB) and valence band (VB) $[9,10]$.

However there are considerable differences in basic physics of HOMO-LUMO as there exists strong Van der Waals forces which are no longer considerable in $\mathrm{CB} / \mathrm{VB}$ formation. Another major differences arises in transport process which is mainly by hopping process between localized states rather than transport within the band. In case of polymers and oligomers, there is hopping along conjugated chain and inter molecular charge transport between adjacent polymer chains or molecules. However the mobility in case of later process is smaller. Thus mobility of thin films can be improved by improving order, purification, high vaccum deposition and no oxygen contamination [11, $12]$.

\section{ORGANIC SOLAR CELL: MECHANISM}

The operating mechanism of organic solar cells are one of the most researched and debated fields. In general all the main differences in mechanism in case of organic solar cell arises due to the generation of electrostatically bound 
electron-hole pair in organic solar cells instead of free charges. Further, this concept is explained in detail.

\subsection{Absorption of Light and Exciton Generation}

The presence of conjugated pi electron system in organic compounds results in all interesting optical and electrical properties. The bandgap or bond energy in organic semiconductors is tuned with the energy of solar spectrum causing the absorption of photons producing electrostatically coupled electron hole pairs called excitons. In case of inorganic silicon semiconductors, produces free charges instead of excitons. This major change drives all the differences in mechanism of electricity generation in inorganic and organic solar photovoltaic devices.

\subsection{Exciton Diffusion}

The photo generated excitons are characterized by very small lifetime of few picoseconds limiting the mobility of excitons to a few polymer units or molecules. The exciton moves within the chain causing chain deformation in order to reduce the extra unstable energy, which is altogether called polaron. However inter-molecular transition of excitons also happens which is termed as hopping process. Altogether the overall mobility of excitons are limited to a range of $10 \mathrm{~nm}$, which is called the exciton diffusion length. As the excitons are to be dissociated within the range of this length, exciton diffusion length plays a critical role in design and performance of organic solar cells.

\subsection{Exciton Dissociation}

Exciton dissociation refers to process of splitting the electrostatically coupled electron hole pair into free charges. The dissociation of excitons occurs at the donor acceptor interfaces or junctions. The donor and acceptor materials are designed such that there exists a difference in LUMO levels of the materials, which drives the exciton dissociation. For efficient dissociation, the difference in energy level of LUMO of donor and acceptor should be higher than that of exciton binding energy. Typically the difference is around $0.2-0.3 \mathrm{eV}$.

In general, to achieve efficient charge separation

\section{$\Delta\left(\mathrm{LUMO}_{\mathrm{D}}-\mathrm{LUMO}_{\mathrm{A}}\right)>$ Exciton binding energy}

\subsection{Charge Transport}

Once the free charges are produced, they travel through specific materials to get collected at the electrodes. From there they are connected to the external circuit. The efficiency of charge transport are determined by the electrical conductivity and impedance of the organic materials [13-16].

\section{RECENT DEVELOPMENTS}

Recent developments in the field of organic solar cell had shown promising results with maximum recorded efficiency over $10 \%$. Overall the researches in the field could be accounted into three sections including study of basic physics and charge transfer dynamics, development of new architectures and novel concepts and materials development for various applications. This section mainly focuses on researches on basic device physics and charge transfer dynamics.

The most important focus is researches are on understanding the working mechanism of organic solar cell. The most primary question of debate is how the bound electron-hole pair splits. The most widely accepted explanation to this question is 'hot exciton effect'. Hot exciton effect describes that when electron is absorbed into one semiconductor material from the other, it carries the differences in energy with it, making the electron hot, gaining velocity with which it escapes from the bounded exciton state. Though this hypothesis is widely accepted recently there has been lots of studies questioning the credibility of the hot exciton hypothesis [17, 18].

Many researchers believe that the electrons that breaks up from the bound state doesn't became hot or gains excess velocity. A lot amount of researches are directed in this direction by spectroscopic studies. Another focus of research is the molecular and intermixed semisolid structure of the semiconductor, where the differences in phase could lead to splitting of excitons [19].

Rather focusing onto various study techniques, it can be generalized that advances in simulation techniques and sophisticated experimental methods are two most promising research tools that are developing onto the study basic physics and charge transport dynamics in organic solar cell operation.

The important computational methodologies that are employed in the study of organic solar cells includes quantum mechanical theories for charge transfer excitations, application to density functional theory (DFT) methods in charge transfer excitations etc. In quantum mechanical simulation of quantum chemistry time dependent density functional theory (TDDFT) has been the most important tool in computations for excited state properties and charge transfer excitations in large systems. [20, 21] Timedependent density functional theory (TDDFT) is a quantum mechanical theory used in chemistry and physics to investigate the dynamics and properties of many-body systems in the presence of time-dependent potentials, such as electric or magnetic fields. The effect of such fields on molecules and solids can be studied with TDDFT to extract features like excitation energies, frequency-dependent response properties, and photo-absorption spectra.Though the implementation of TDDFT calculations on to photovoltaic systems possesses a number of challenges particularly with respect to long range charge transfer interactions. The effect of assumptions in this applications possibly results in catastrophic errors in simulations. Many theories and improvements have been suggested to this basic approach to enhance the results of simulation [22, 23]. 
Another method is constraint variational approach including constrained DFT (CDFT), which effectively overcomes many drawbacks in the TDDFT approach. CDFT is mainly applicable to systems whose ground state electron density satisfies some specific constraints. In some electron transfer reactions, the constraint amount to localization of the electronic density such that the difference in the number of electrons between donor and acceptor is reduced by two compared to that of the unconstrained ground state [24-28].

The other widely used methodology in simulation study includes the applications of DFT methods in CT excitations. Density functional theory (DFT) is a computational quantum mechanical modelling method used in chemistry, physics and materials science to investigate the electronic structure (principally the ground state) of many-body systems, in particular atoms, molecules, and the condensed phases. With this theory, the properties of a many-electron system can be determined by using functionals, i.e. functions of another function. DFT based computational methods can be used to calculate or develop structure function relationships for many properties of importance in organic photovoltaics including bandgap, optical absorption, intra-molecular and inter-molecular charge transfer, exciton binding energy, charge transfer integral, reorganization energy and the rate of charge transfer and recombination in donor-acceptor complexes. In practical systems, the effects of the surrounding organic photovoltaics media on these properties should be taken into account. Although the effect of medium depends both on its nature and on that of the transitions involved, at least solvation can be routinely taken into account at reasonable computational cost by considering polarization continuum solvation models [29, 30].

The direction of experimental research going on this field primarily accounts onto spectroscopic analysis of the processes involved in the conversion processes. It possess many challenges on obtaining a detailed description as the processes happens within a time of few picoseconds. As pointed an important controversial statement in the field is that the main focus of this research have been towards development and study of semiconductors which are capable of extracting the energy by making hot electrons. The out of equation on this point demands for the change of focus from developing semiconductors that extract hot electrons to semiconductors which are capable of extracting energy out of it without the electrons being hot. It suggest towards a fundamental shift in the organic photovoltaics research [31, 32].

\section{CONCLUSION}

Organic solar cells shows good promises in the development of low cost photovoltaic alternatives. A structured and systematic research are required in the field to successfully utilize the foreseen advantages of organic solar cells.

The review on basic physics, charge transfer dynamics, mechanism and recent approaches and developments in the field are carried out. It is very important to understand the basic mechanism of operation in conversion of light into electricity. Since all the design, architecture and materials developments revolve around our level of understanding of the basic mechanism it accounts chief importance in all researches going on in the field of organic photovoltaics.

\section{REFERENCES}

[1]. A. Hagfeldt, G. Boschloo, L. Sun, L. Kloo, and H. Pettersson, "Dye-sensitized solar cells.," Chem. Rev., vol. 110, pp. 6595-6663, 2010.

[2]. A. . Nozik, "Quantum dot solar cells," Physica E: Lowdimensional Systems and Nanostructures, vol. 14. pp. 115120, 2002.

[3]. G. Chamberlain, "Organic solar cells: A review," Sol. Cells, vol. 8, pp. 47-83, 1983.

[4]. J. You, L. Dou, K. Yoshimura, T. Kato, K. Ohya, T. Moriarty, K. Emery, C.-C. Chen, J. Gao, G. Li, and Y. Yang, "A polymer tandem solar cell with $10.6 \%$ power conversion efficiency.," Nat. Commun., vol. 4, p. 1446, Jan. 2013.

[5]. H. Hoppe and N. S. Sariciftci, "Organic solar cells: An overview," Journal of Materials Research, vol. 19. pp. 1924-1945, 2004.

[6]. J. Nelson, "Organic photovoltaic films," Curr. Opin. Solid State Mater. Sci., vol. 6, pp. 87-95, 2002.

[7]. J.-L. Brédas, J. E. Norton, J. Cornil, and V. Coropceanu, "Molecular understanding of organic solar cells: the challenges.," Acc. Chem. Res., vol. 42, pp. 1691-1699, 2009.

[8]. H. Shirakawa, E. J. Louis, A. G. MacDiarmid, C. K. Chiang, and A. J. Heeger, "Synthesis of electrically conducting organic polymers: halogen derivatives of polyacetylene, $(\mathrm{CH}) \mathrm{x}$," Journal of the Chemical Society, Chemical Communications. p. 578, 1977.

[9]. W. Brütting, "Organic Semiconductors," Semiconductors, vol. 6, pp. 1-11, 2005.

[10]. G. Chidichimo and L. Filippelli, "Organic solar cells: Problems and perspectives," Int. J. Photoenergy, vol. 2010, 2010.

[11]. S. Cowan and W. Leong, "a Threshold Impurity Level for Organic Solar Cells: Enhanced First- Order Recombination Via Well- Defined PC84BM Traps in Organic Bulk Heterojunction Solar Cells," Adv. Funct. ..., vol. 21, p. n/a-n/a, 2011

[12]. G. Heimel, I. Salzmann, S. Duhm, and N. Koch, "Design of organic semiconductors from molecular electrostatics," Chem. Mater., vol. 23, pp. 359-377, 2011

[13]. R. C. Chiechi, R. W. A. Havenith, J. C. Hummelen, L. J. A. Koster, and M. A. Loi, "Modern plastic solar cells: Materials, mechanisms and modeling," Materials Today, vol. 16. pp. 281-289, 2013.

[14]. H. Bässler and A. Köhler, "Charge transport in organic semiconductors," Top. Curr. Chem., vol. 312, pp. 1-65, 2012.

[15]. X. Y. Zhu, Q. Yang, and M. Muntwiler, "Chargetransfer excitons at organic semiconductor surfaces and interfaces," Acc. Chem. Res., vol. 42, pp. 1779-1787, 2009. [16]. G.-J. a H. Wetzelaer and P. W. M. Blom, "Diffusiondriven currents in organic-semiconductor diodes," NPG Asia Mater., vol. 6, no. 7, p. e110, Jul. 2014. 
[17]. S. Tiwari and N. C. Greenham, "Charge mobility measurement techniques in organic semiconductors," Opt. Quantum Electron., vol. 41, pp. 69-89, 2009.

[18]. J. M. Lee, B. H. Kwon, H. Il Park, H. Kim, M. G. Kim, J. S. Park, E. S. Kim, S. Yoo, D. Y. Jeon, and S. O. Kim, "Exciton dissociation and charge-transport enhancement in organic solar cells with quantum-dot/N-doped CNT hybrid nanomaterials," Adv. Mater., vol. 25, pp. 2011-2017, 2013.

[19]. Mark Shwartz, "Stanford study could lead to paradigm shift in organic solar cell research," Stanford Report, 2013. [Online]. Available: http://news.stanford.edu/news/2013/november/excitonsolar-cell-111913.html. [Accessed: 20-Aug-2014]. .

[20]. K. Burke, J. Werschnik, E.K.U. Gross, "Time dependent density functional theory: past, present and future", The Journal of Chemical Physics 123 (2005) 062206.

[21]. J. Autschbach, "Charge transfer excitations and time dependent desity functional theory: problems and some proposed solutions", ChemPhysChem 10, 2009, 1757-1760.

[22]. S. Grimme, F. Neese, "Double hybrid density functional theory for excited electronic states of molecules", The Journal of Chemical Physics 127, 2007, 154116.

[23]. Y. Zhao, D. G. Truhlar, "Density functionals with broad applicability in chemistry", Accounts of Chemical Research 41, 2008, 157-167.

[24]. Q. Wu, T. Van Voorhis, "Direct calculation of electron transfer parameters through constrained density functional theory", The Journal of Physical Chemistry A 110, 2006, 9212-9218.

[25]. Q. Wu, T. V. Voohris, "Extracting electron transfer coupling elementsfrom constrained density functional theory", The Journal of Chemical Physics 125, 2006, 164105.

[26]. T. Van Voohris, T. Kowalczyk, B. Kaduk, L. P. Wang, C. L. Cheng, Q. Wu, "The diabatic picture of electron transfer, reaction barriers, and molecular dynamics", Annual review of Physical Chemistry 61, 2010, 149-170.

[27]. S. Difley, L. P. Wang, S. Yeganesh, S. R. Yost, T. V. Voohris, "Electronic properties of disordered organic semicponductors via QM/MM simulations", Accounts of Chemical Research 43, 2010, 995-1004.

[28]. Q. Wu, T. V. Voohris, "Direct optimization method to study constrained systems within density functional theory", Physical Review A 72, 2005, 024502.

[29]. M. Segal, M. Singh, K. Revoire, S. Difley, T. V. Voohris, M. A. Baldo, "Extra fluorescent electro luminescence in organic light emitting devices, Nature Materials 6, 2007, 374-378.

[30]. S. Difley, T. V. Voohris, "Exciton/Charge transfer electronic couplings in organic semiconductors", Journal of Chemical Theory and Compuitation 7, 2011, 594-601.

[31]. "Recombination dynamics in Organic solar cells", Dissertation zur Erlangung des Alexander Fortig im Odenwald, 2013. 